$\underline{\text { www.scielo.br/eq }}$

\title{
Oxidação eletroquímica do metanol sobre partículas de PtRu e PtMo suportadas em carbono de alta área superficial
}

\author{
A. Oliveira Neto ${ }^{1}$, M. Linardi ${ }^{1}$, E. R. Gonzalez ${ }^{2}$ \\ ${ }^{1}$ Instituto de Pesquisa Energéticas e Nucleares- Centro de Ciência e Tecnologia de Materiais- Laboratório de Células a \\ Combustível PEM - SP - Brasil \\ ${ }^{2}$ Instituto de Química de São Carlos - USP - CEP 13560-970 - São Carlos - SP - Brasil
}

Resumo: A reação de eletro-oxidação do metanol foi estudada sobre eletrocatalisadores de Pt/C, PtRu/ $\mathrm{C}$ e $\mathrm{PtMo} / \mathrm{C}$ preparados pelo método do ácido fórmico em diferentes composições atômicas.

Os produtos da oxidação do metanol foram monitorados pela técnica de DEMS.

O desempenho dos catalisadores frente a reação de oxidação do metanol foi estudado através dos perfis voltamétricos e experimentos de cronoamperoometria.

Palavras-chave: eletrocatalisadores; oxidação do metanol; células a combustível

\section{Introdução}

O mecanismo da oxidação de metanol foi revisado por Parsons e Vandernoot em 1988 [1] e por Hamnett [2,3] em 1997, e apresenta dois aspectos fundamentais: 1) a eletrossorção de metanol e 2) a adição de oxigênio ao intermediário contendo carbono para gerar $\mathrm{CO}_{2}$.

Poucos são os materiais sobre os quais o metanol se adsorve. Em solução ácida só platina [318] e ligas de platina [19-36] apresentam atividade sensível à oxidação do metanol e estabilidade em condições operacionais. Esta é a razão principal para que quase todos os estudos mecanísticos estejam concentrados sobre estes materiais.

A existência de vários intermediários na oxidação do metanol é de consenso de diversos pesquisadores, entretanto ainda não está completamente esclarecido o mecanismo pelo qual esta oxidação ocorre. A seguir apresenta-se as possíveis etapas aceitas para a oxidação de metanol sobre platina $[2,37]$.

$$
\begin{aligned}
& \mathrm{CH}_{3} \mathrm{OH}+\mathrm{Pt}_{(\mathrm{s})} \rightarrow \mathrm{Pt}-\mathrm{CH}_{2} \mathrm{OH}_{\text {ads }}+\mathrm{H}^{+}+\mathrm{e}^{-} \\
& \mathrm{Pt}_{-} \mathrm{CH}_{2} \mathrm{OH}_{\text {ads }}+\mathrm{Pt}_{(\mathrm{s})} \rightarrow \mathrm{Pt}_{2}-\mathrm{CHOH}_{\text {ads }}+\mathrm{H}^{+}+\mathrm{e}^{-} \\
& \mathrm{Pt}_{2}-\mathrm{CHOH}_{\text {ads }}+\mathrm{Pt}(\mathrm{s}) \rightarrow \mathrm{Pt}_{3}-\mathrm{COH}_{\text {ads }}+\mathrm{H}^{+}+\mathrm{e}^{-} \\
& \mathrm{Pt}_{3}-\mathrm{COH}_{\text {ads }} \rightarrow \mathrm{Pt}-\mathrm{CO}_{\text {ads }}+2 \mathrm{Pt}(\mathrm{s})+\mathrm{H}^{+}+\mathrm{e}^{-} \\
& \mathrm{Pt}(\mathrm{s})+\mathrm{H}_{2} \mathrm{O} \rightarrow \mathrm{Pt}^{-} \mathrm{OH}_{\mathrm{ads}}+\mathrm{H}^{+}+\mathrm{e}^{-} \\
& \mathrm{Pt}-\mathrm{CO}_{\text {ads }}+\mathrm{H}_{2} \mathrm{O} \rightarrow \mathrm{Pt}-\mathrm{COOH}_{\text {ads }}+\mathrm{H}^{+}+\mathrm{e}^{-}
\end{aligned}
$$

ou

$$
\begin{aligned}
& \mathrm{PtOH}_{\text {ads }}+\mathrm{Pt}_{-} \mathrm{CO}_{\text {ads }} \rightarrow \mathrm{Pt}-\mathrm{COOH}_{\text {ads }} \\
& \mathrm{Pt}-\mathrm{COOH}_{\text {ads }} \rightarrow \mathrm{Pt}_{(\mathrm{s})}+\mathrm{CO}_{2}+\mathrm{H}^{+}+\mathrm{e}^{-}
\end{aligned}
$$

Observa-se que as reações 1-3 são processos de eletrossorção enquanto as reações 
seguintes envolvem transferência de oxigênio para a oxidação dos intermediários ligados à superfície [2].

Os itens mais relevantes a respeito do mecanismo da oxidação de metanol são: 1) a adsorção do metanol sobre sítios favorecidos energeticamente na superfície, 2) a perda seqüencial dos prótons para dar origem a uma sequiência de intermediários hidrogenados (de tempos de vida curtos) multiplamente ligados que se convertem em $\mathrm{CO}$ adsorvido linearmente. Esta evidência provém dos resultados obtidos por FTIR [38,39], DEMS $[40,41]$ e estudos dos produtos da reação [42] e 3) a análise cinética sugere que o mecanismo para a oxidação de metanol sobre platina envolve o ataque de $\mathrm{H}_{2} \mathrm{O}$ sobre a molécula de $\mathrm{CO}$. Acima de $0,6 \mathrm{~V}$ vs ERH o CO é oxidado rapidamente na superfície da platina e dados de FTIR sugerem que intermediários como $\mathrm{Pt}_{3} \mathrm{COH}$ podem oxidar-se diretamente $\mathrm{CO}_{2}$ em altos potenciais.

Devido a seu envenenamento, a platina sozinha não é suficientemente ativa para ser usada comercialmente na oxidação do metanol. Pode-se usar a platina combinada com óxidos metálicos como o $\mathrm{WO}_{3}$ [43-44] e uma outra alternativa é o uso de catalisadores bifuncionais onde o segundo metal (menos nobre que a Pt) como Ru [21-28], Mo [35,45], Sn [20] fornece o oxigênio de forma ativa, facilitando a oxidação dos intermediários.

Muitos dos trabalhos empregam ligas de platina-rutênio, pois estas têm se mostrado, excelentes eletrocatalisadores em condições operacionais [21-28].

A reação de oxidação de metanol ocorre sobre a superfície de Pt (ou modificada com rutênio) e passa por diferentes etapas: para valores menores de 0,35 V vs. ERH, sobre Pt (111) a adsorção de metanol é parcialmente inibida por hidrogênio adsorvido. Em potenciais mais altos (na região da dupla camada) o metanol pode deslocar moléculas de água para chegar a ser adsorvido fisicamente, antes de quimioadsorver-se. Para valores acima de $0,6 \mathrm{~V}$ a dissociação da água produz $\mathrm{OH}_{\text {ads }}$, outro inibidor da adsorção de metanol. A espécie $\mathrm{OH}_{\text {ads }}$ facilita a oxidação de $\mathrm{CO}$ adsorvido mas inibe a adsorção de metanol, portanto desempenha um papel duplo.

Na presença de rutênio, em valores de potencial acima de $0,2 \mathrm{~V}$ vs. ERH ocorre a formação de espécies oxigenadas que modificam as etapas anteriormente mencionadas. No intervalo de potenciais entre 0,35 e $0,6 \mathrm{~V}$ para a oxidação de metanol sobre ligas de Ru, Iwasita et al. [21] propõem o seguinte mecanismo bifuncional:

$$
\begin{aligned}
& \mathrm{CH}_{3} \mathrm{OH}+\mathrm{Pt}\left(\mathrm{H}_{2} \mathrm{O}\right) \rightarrow \mathrm{Pt}\left(\mathrm{CH}_{3} \mathrm{OH}\right)_{\text {ads }}+\mathrm{H}_{2} \mathrm{O} \\
& \mathrm{Pt}\left(\mathrm{CH}_{3} \mathrm{OH}\right)_{\mathrm{ads}} \rightarrow \mathrm{Pt}(\mathrm{CO})_{\text {ads }}+4 \mathrm{H}^{+}+4 \mathrm{e}^{-} \\
& \mathrm{Ru}\left(\mathrm{H}_{2} \mathrm{O}\right) \rightarrow \mathrm{RuOH}+\mathrm{H}^{+}+\mathrm{e}^{-} \\
& \mathrm{Pt}(\mathrm{CO})_{\mathrm{ads}}+\mathrm{RuOH} \rightarrow \mathrm{Pt}+\mathrm{Ru}+\mathrm{CO}_{2}+\mathrm{H}^{+}+\mathrm{e}^{-} \\
& \mathrm{Ru}(\mathrm{CO})_{\mathrm{ads}}+\mathrm{RuOH} \rightarrow 2 \mathrm{Ru}+\mathrm{CO}_{2}+\mathrm{H}^{+}+\mathrm{e}^{-}
\end{aligned}
$$

Aqui é admitida a adsorção de metanol na equação (8) como uma troca de uma molécula de água adsorvida por uma molécula de metanol. A reação passa por várias etapas e requer a presença de sítios vizinhos de Pt para facilitar a dissociação do metanol.

No intervalo de potenciais de $0,35-0,6 \mathrm{~V}$ a reação (8) não é a etapa determinante no caso de existir suficientes átomos de Pt nos sítios vizinhos. Acima de $0,7 \mathrm{~V}$ a adsorção de água começa a ser mais forte que a fisissorção do metanol. Este fato favorece a formação de $\mathrm{Pt}(\mathrm{OH})_{\text {ads }}$, diminuindo a velocidade de reação. Por outro lado, na reação (9), a dissociação de metanol para formar CO (extendido a outros adsorbatos) pode acontecer a potenciais relativamente baixos. Para a Pt isto acontece em $0,15 \mathrm{~V}$ tendo um máximo em 0,25 V. Na presença de $\mathrm{Ru}$ eletrodepositado, a adsorção dissociativa do metanol é deslocada $0,05 \mathrm{~V}$ para valores menos positivos, o que torna difícil assinalar esta reação como a etapa determinante da reação no intervalo de potenciais entre 0,35 e $0,6 \mathrm{~V}$, a não ser que existam insuficientes átomos de platina nos sítios vizinhos.

Visando a parte aplicativa, é de muito interesse determinar a relação ótima de platinarutênio para a oxidação de metanol Iwasita el al. [21] mostram que para ligas de platina-rutênio com composições superficiais entre 10 e $40 \%$ de Ru, a reação é independente do conteúdo de Ru. No estudo por eles realizado conclui-se que a reação entre o $\mathrm{CO}_{\text {ads }}$ e o $\mathrm{OH}$ deve ser a responsável pela velocidade do processo no intervalo de potenciais entre 0,35 e $0,6 \mathrm{~V}$. 


\section{Procedimento experimental}

Os catalisadores de platina dispersa, platina-rutênio e platina-molibdênio foram preparados pelo método do ácido fórmico [45]. Os resultados eletroquímicos apresentados neste trabalho foram obtidos através das técnicas de voltametria cíclica, DEMS (espectrometria de massa diferencial eletroquímica) e cronoamperometria.

O DEMS nos dá informações a respeito dos produtos e do mecanismo da reação de oxidação do metanol [21].

As medidas de voltametria cíclica foram realizadas com um potenciostato/galvanostato da EGG Princeton Applied Research Modelo 273A acoplado a um micro computador IBM-PC.

Para os experimentos de voltametria cíclica utilizou-se uma célula convencional de um compartimento com o corpo confeccionado em vidro pyrex. Esta célula possui orifícios para o eletrodo de referência, contra eletrodo, eletrodo de trabalho e mangueira de fluxo de gás. O eletrodo de referência usado foi o eletrodo reversível de hidrogênio (ERH), o contra eletrodo constituí-se de uma placa de platina soldada a um fio de platina. Para a confecção do eletrodo de trabalho, um cilindro de grafite pirolítico $\left(0,196 \mathrm{~cm}^{2}\right)$ foi moldado em Teflon de tal modo que pudesse ser adaptado a um suporte especialmente construído para se adaptar ao sistema de eletrodo de disco rotatório. No cilindro de grafite foi feita uma cavidade $(0,3 \mathrm{~mm}$ de profundidade $)$ para a colocação da camada ativa do catalisador disperso[45].

Os voltamogramas cíclicos foram registrados em soluções de $0,5 \mathrm{~mol} \mathrm{~L}^{-1}$ de $\mathrm{H}_{2} \mathrm{SO}_{4}$ desaeradas com nitrogênio na presença e ausência de metanol. Durante o experimento as soluções foram mantidas sob atmosfera de nitrogênio. A velocidade de varredura de potencial foi de $10 \mathrm{mV} / \mathrm{s}$. Os limites catódico e anódico foram delimitados pela descarga de hidrogênio e oxigênio respectivamente.

Para os experimentos de cronoamperometria fixou-se um valor de potencial de forma instantânea, com o auxílio de um potenciostato e observou-se o comportamento da corrente em função do tempo.

Após a realização de alguns experimentostestes, chegou-se a um intervalo de tempo de 1800 s para que houvesse uma estabilização no valor da corrente. Os valores lidos em 1800s foram então utilizados para a construção das curvas I x E. As medidas de cronoamperometria foram realizadas em soluções de $0,5 \mathrm{~mol} \mathrm{~L}^{-1}$ de $\mathrm{H}_{2} \mathrm{SO}_{4}$ na presença de $0,1 \mathrm{~mol} \mathrm{~L}^{-1}$ de metanol para os catalisadores de platina dispersa, liga de platina-rutênio e liga de platina-molibdênio.

As ligas de platina-rutênio e platinamolibdênio foram preparadas nas composições atômicas (60:40), (70:30), (80:20) e (90:10).

\section{Resultados e discussão}

O catalisador E-TEK apresenta uma composição diferente aos demais catalisadores, além de ser preparado por um método distinto aos demais catalisadores, por isso não se pode comparar diretamente os resultados apresentados, mas poderíamos obter informações quanto a otimização da composição de catalisadores PtRu para a oxidação do metanol.

A Figura 1 apresenta a comparação de corrente com relação ao potencial para os catalisadores platina-rutênio preparados pelo método do ácido fórmico e catalisador comercial E-TEK a $10 \mathrm{mV} \mathrm{s}^{-1}$ considerando-se apenas a varredura anódica.

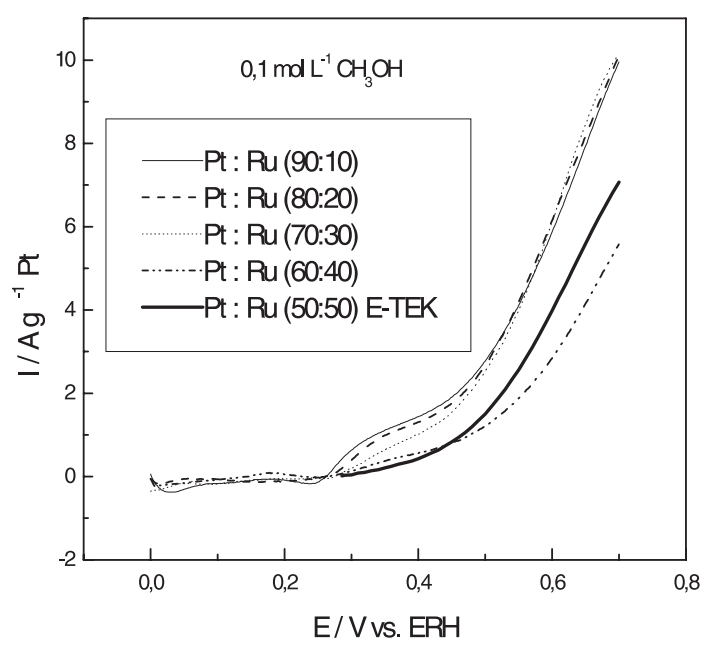

Figura 1. Comparação das correntes dos catalisadores de platina rutênio preparados pelo método do ácido fórmico e catalisador comercial E-TEK a $10 \mathrm{mVs}^{-1}$ considerando-se

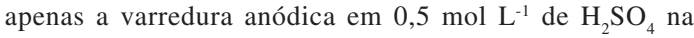
presença de metanol $0,1 \mathrm{~mol} \mathrm{~L}^{-1}$. 
As ligas de platinas com 10\%, 20\% e 30\% de $\mathrm{Ru}$ apresentam uma maior atividade catalítica com relação aos outros catalisadores em toda a faixa de potencial estudada. Considerando a faixa de potencial para aplicações tecnológicas $(0,4$ a 0,5 V) podemos dizer que as ligas com 10 e $20 \%$ de $\mathrm{Ru}$ apresentam-se mais ativas para a oxidação do metanol. Podemos dizer que o melhor catalisador é aquele que apresenta um início da oxidação em menores potenciais possíveis.

O catalisador com $40 \%$ Ru apresenta um pior desempenho com relação aos demais catalisadores de rutênio preparados neste laboratório em toda a faixa de potencial estudada, mas este apresenta uma atividade catalítica superior ao catalisador comercial da E-TEK até potenciais de $0,45 \mathrm{~V}$.

Acima de $0,5 \mathrm{~V}$ o catalisador da E-TEK apresenta melhor desempenho em relação ao catalisador PtRu (60:40) preparado pelo método do ácido fórmico.

Os resultados da Figura 1 estão em acordo com Gasteiger et al. ${ }^{[13]}$ onde ligas com $7 \%$ de Ru foram as mais ativas para a eletro-oxidação do metanol. Isto ocorre porque há um número máximo de átomos de platina necessários para adsorção do metanol enquanto o rutênio fornece espécies $\mathrm{OH}$ em sítios adjacentes. Estes autores atribuem a alta atividade observada para a liga com $7 \%$ de $\mathrm{Ru}$ ao requerimento de átomos de platina que facilitem a adsorção dissociativa do metanol.

Em acordo com a discussão apresentada acima, Richarz et al. [29] também sugerem que as melhores ligas para a oxidação do metanol são as ligas com 7-30\%. Por outro lado, para a oxidação monóxido de carbono os melhores resultados se observam com a liga com 50\% de Ru [29].

Souza et al. [46] mostram que a ordem de atividade na região de potencial de 0,4 a $0,7 \mathrm{~V}$ é: platina-rutênio $(75: 25)>$ platina-rutênio $(86: 14)=$ platina-rutênio (65:35)> platina.

Na Figura 2 são apresentadas as curvas de corrente com relação ao potencial (varredura anódica), para os diferentes catalisadores de platina-molibdênio preparados neste laboratório e para o catalisador comercial da E-TEK. O catalisador da E-TEK é uma liga de platinamolibdênio (75:25), 30\% em peso disperso em carbono de alta área superficial, enquanto que os catalisadores de platina-molibdênio preparados neste trabalho possuem $20 \%$ em peso.

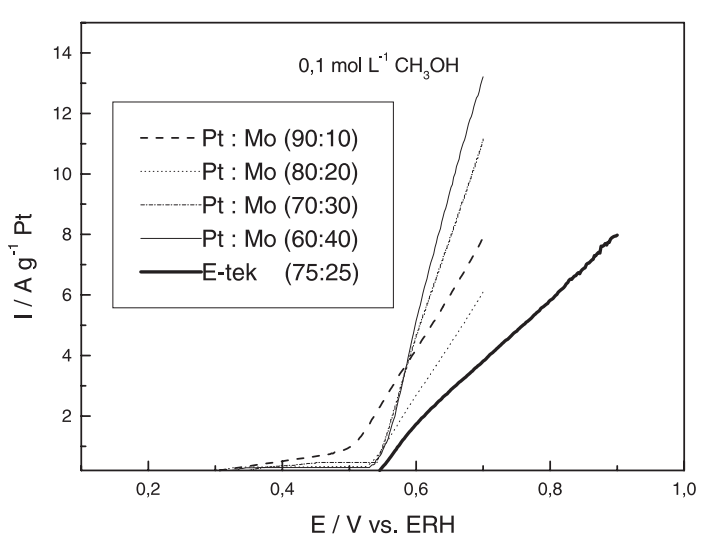

Figura 2. Comparação das curvas de corrente vs. potencial para os diferentes catalisadores de platina-molibdênio preparados neste laboratório e o catalisador comercial da ETEK, em $0,5 \mathrm{~mol} \mathrm{~L}^{-1} \mathrm{H}_{2} \mathrm{SO}_{4}$ na presença de $0,1 \mathrm{~mol} \mathrm{~L}^{-1}$ considerando-se apenas a varredura anódica.

O início da oxidação do metanol ocorre em menores potenciais para a liga de platinamolibdênio (90:10), demonstrando uma alta atividade catalítica desta liga com relação às outras, porém esta liga apresenta um pior desempenho quando comparada com a liga platina-molibdênio (60:40) em altos potenciais. Para potenciais acima de $0,58 \mathrm{~V}$ podemos classificar os catalisadores quanto às suas correntes como segue:

$$
\mathrm{I}_{60: 40}>\mathrm{I}_{70: 30}>\mathrm{I}_{90: 10}>\mathrm{I}_{80: 20}>\mathrm{I}_{\mathrm{E}-\mathrm{TEK}} .
$$

Todos os catalisadores preparados neste laboratório, no intervalo de potencial estudado, apresentaram maiores valores de corrente e um maior efeito catalítico com relação ao catalisador comercial. O melhor desempenho observado para a liga de platina-molibdênio (90:10) com relação a liga de platina-molibdênio (80:20), pode estar associado a uma melhor dispersão deste catalisador no suporte, ou seja, este catalisador poderia apresentar uma maior área superficial disponível para a reação. Uma outra hipótese é que a liga de platina-molibdênio (90:10) apresenta pequena quantidade de molibdênio, podendo apresentar um comportamento bastante similar ao da platina 
dispersa, sendo que isto poderia refletir no melhor desempenho observado na figura 2 .

A Figura 3 mostra uma comparação dos resultados de voltametria cíclica a $10 \mathrm{mV} \mathrm{s}^{-1}$ considerando-se apenas a varredura anódica para as ligas platina-molibdênio (60:40), platina-rutênio (80:20) e platina dispersa em $0,5 \mathrm{~mol} \mathrm{~L}^{-1} \mathrm{H}_{2} \mathrm{SO}_{4}$ na presença de $0,1 \mathrm{~mol} \mathrm{~L}^{-1}$ de metanol. A liga de platina-molibdênio (60:40) apresenta um efeito de corrente apreciável em potenciais mais altos com relação aos outros catalisadores de platinamolibdênio, por isso a sua escolha para a comparação. Os resultados apresentados neste trabalho mostram que ligas de platina-rutênio com composições atômicas entre $10 \%$ e $30 \%$ de Ru apresentam um melhor desempenho para a eletrooxidação do metanol. Para a comparação dos resultados, a liga de platina-rutênio $(80: 20)$ foi escolhida.

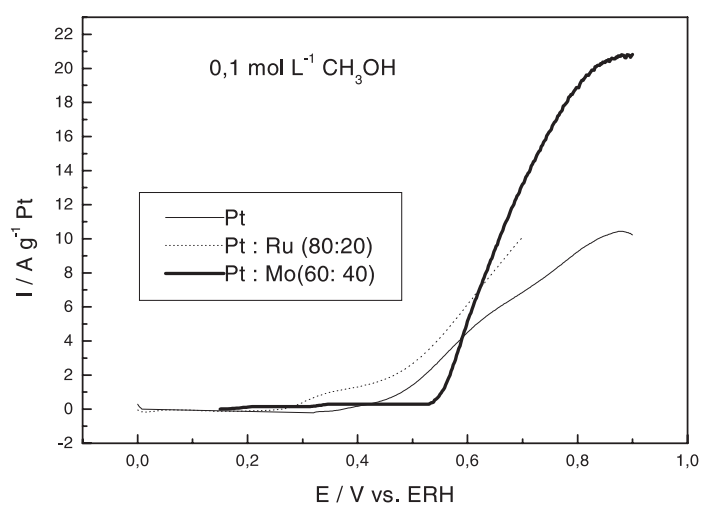

Figura 3. Comparação dos catalisadores platina-molibdênio (60:40), catalisador platina-rutênio (80:20) e platina dispersa em $0,5 \mathrm{~mol} \mathrm{~L}^{-1} \mathrm{H}_{2} \mathrm{SO}_{4}$ na presença de $0,1 \mathrm{~mol} \mathrm{~L}^{-1}$ de metanol a $10 \mathrm{mV} \mathrm{s}^{-1}$ considerando-se apenas a varredura anódica.

A eletro-oxidação do metanol ocorre em potenciais mais baixos para a liga de platinarutênio (80:20), seguida pela platina dispersa e por último pela liga de platina-molibdênio (60:40). Para potencias acima de $0,6 \mathrm{~V}$ a liga de platina-molibdênio (60:40) apresenta melhores níveis de corrente seguida pela liga de platinarutênio (80:20) e pela platina dispersa.

As ligas de platina-molibdênio não são efetivas para dar início à eletro-oxidação do metanol, contudo o aumento da corrente na eletrooxidação do metanol denota uma melhor tolerância às espécies fortemente adsorvidas formadas no processo, ou seja, as ligas de platinamolibdênio apresentam uma alta atividade para a oxidação de CO. A alta atividade para a oxidação de metanol se deve à contínua oxidação do CO e subsequiente liberação de sítios de platina que são requeridos para adsorção dissociativa do metanol.

Levando-se também em consideração os estudos para diferentes catalisadores em células à combustível alimentadas diretamente com metanol, tem-se observado que ligas de platina-rutênio apresentam um melhor desempenho com relação aos demais catalisadores e com isso criou-se uma grande expectativa quanto à sua utilização nestas células. Gotz et al. ${ }^{[35]}$ realizaram estudos em células a combustível de metanol direto utilizando catalisadores à base de platina-rutênio. Estes estudos mostram um forte efeito catalítico das ligas de platina-rutênio, seguido pelas ligas de platina-tungstênio e platina-molibdênio. A adição de estanho diminui a atividade catalítica comparada com o catalisador de platina dispersa. No caso dos sistemas ternários a base de platinarutênio preparada pelo método de impregnação, platina-rutênio-tungstênio é o sistema mais ativo para a oxidação do metanol, seguido por platinarutênio-molibdênio e platina-rutênio. O sistema platina-rutênio-estanho é menos ativo que o sistema platina-rutênio. O catalisador E-TEK é mais ativo que os catalisadores ternários e o de platina-rutênio preparado pelo método de impregnação. Isto pode ser explicado pelo fato que a liga de platina-rutênio E-TEK apresenta uma liga mais uniforme, enquanto que o catalisador preparado pelo método de impregnação apresenta estrutura cúbica de face centrada para a platina e estrutura hexagonal para o rutênio [37].

Os sinais de massa analisados neste trabalho foram massa $44\left(\mathrm{CO}_{2}\right)$, e massa 60 $\left(\mathrm{HCOOCH}_{3}\right)$, os sinais de $\mathrm{m} / \mathrm{z}=44$ são maiores do que os sinais de $\mathrm{m} / \mathrm{z}=60$, indicando que o produto mais importante da eletro-oxidação do metanol sobre platina, platina-rutênio e platina-molibdênio é o $\mathrm{CO}_{2}$. A Figura 4 mostra uma comparação da intensidade de massa 44 normalizada para os catalisadores investigados. 


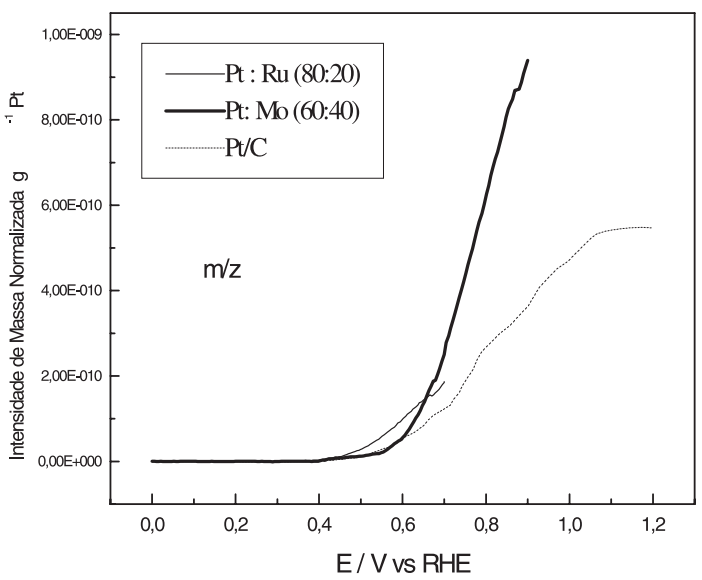

Figura 4. Comparação dos sinais de massa on-line normalizados vs. potencial para a eletro-oxidação do metanol utilizando a técnica de DEMS para o catalisador de platinarutênio (80:20), catalisador de platina-molibdênio(60:40) e catalisador de platina dispersa em $0,5 \mathrm{~mol} \mathrm{~L}^{-1} \mathrm{de}_{2} \mathrm{SO}_{4}$ na presença de metanol $0,1 \mathrm{~mol} \mathrm{~L}^{-1}$.

A liga de platina-rutênio (80:20) começa a produzir massa $44 \mathrm{em}$ menores potenciais com relação aos catalisadores de platina e platinamolibdênio (60:40), mas acima do potencial de $0,65 \mathrm{~V}$ a produção de massa 44 sobre o catalisador de platina-molibdênio (60:40) é muito maior com relação aos catalisadores de platina e platinarutênio. O catalisador de platina-molibdênio (60:40) não é muito eficiente para o início da formação da massa 44, mas em potenciais mais altos apresenta um excelente desempenho. A formação de massa $44 \mathrm{em}$ menores potenciais para a liga de platina-rutênio com relação a platina dispersa está de acordo com os trabalhos da literatura [46].

Com a técnica da voltametria cíclica podese ter informações à respeito do comportamento da superfície do eletrodo na solução. Após terem sido feitas medidas voltamétricas com velocidades de varredura de $10 \mathrm{~m} \mathrm{V/s}$, optou-se por obter os valores de corrente-potencial utilizando-se a técnica de cronoamperometria. $\mathrm{Na}$ técnica de cronoamperometria quando uma pertubação no potencial é aplicada além do fator tempo a ser considerado devemos considerar os efeitos de transporte de massa e a possibilidade de formação de bolhas $\left(\mathrm{de}_{2} \mathrm{CO}_{2}\right)$ sobre a superfície do eletrodo.

As curvas de corrente vs potencial construídas a partir dos resultados de cronoamperometria em 1800 segundos para os catalisadores de platina-molibdênio (60:40), platina-rutênio (80:20) e platina dispersa são apresentadas na Figura 5.

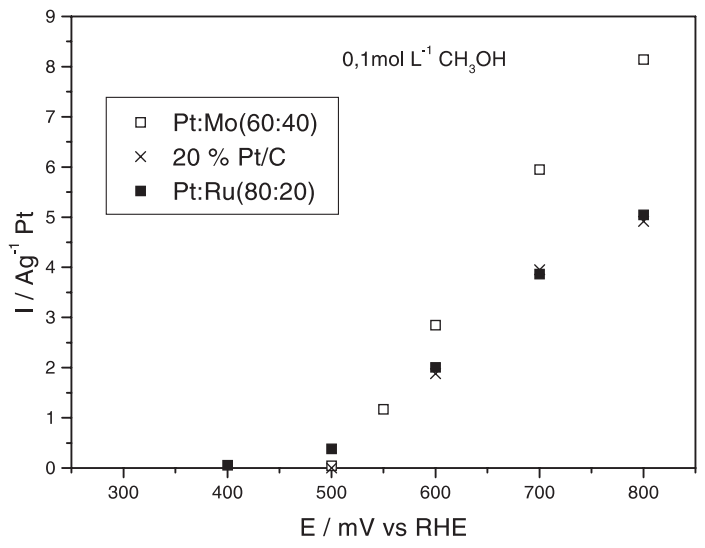

Figura 5. Comparação das curvas corrente-potencial construídas a partir dos resultados de cronoamperometria para o catalisador de platina-molibdênio (60:40), de platina-rutênio (80:20) e de platina dispersa na presença de $0,5 \mathrm{~mol} \mathrm{~L}^{-1}$ de $\mathrm{H}_{2} \mathrm{SO}_{4}+0,1 \mathrm{~mol} \mathrm{~L}^{-1}$ de metanol.

Os resultados apresentados Figura 5 mostram que a eletro-oxidação do metanol ocorre em menores potenciais para o catalisador de platina-rutênio (80:20), mas em potenciais acima de $0,6 \mathrm{~V}$ o catalisador de platina-molibdênio (60:40) apresenta maiores valores de corrente. Também se observa na Figura 5 que a liga de platina-rutênio (80:20) apresenta maior valor de corrente em 0,5 $\mathrm{V}$ com relação à platina dispersa, sendo este resultado em acordo com a referência [26].

Os resultados de cronoamperometria (Figura 5) estão de acordo com os resultados de DEMS (Figura 4) e voltametria cíclica (Figura 3), onde o mesmo comportamento para os catalisadores em estudo é observado. Estes resultados mostram o efeito benéfico da adição de co-catalisadores a platina, principalmente a adição de rutênio a platina.

\section{Conclusões}

O método de preparação de catalisadores desenvolvido neste laboratório permitiu a obtenção de materiais ativos para a eletro-oxidação do metanol. 
O resultado para a platina dispersa, liga de platinarutênio (80:20) e platina-molibdênio (60:40) frente à oxidação do metanol utilizando as técnicas de voltametria cíclica, DEMS e cronoamperometria, indicam que catalisadores de platina-rutênio são efetivos para o início da oxidação do metanol e para o potencial de interesse das células a combustível. Os catalisadores de platinamolibdênio apresentam valores de correntes apreciáveis em altos potenciais, os quais não são de interesse para as aplicações em células a combustível.

De um ponto de vista teórico, as ordens de atividade catalítica obtidas em relação à composição dos catalisadores de platina-rutênio apontam para uma interpretação via mecanismo bifuncional para a ação promotora do rutênio nos catalisadores de platina-rutênio.

Quanto ao mecanismo para a eletro-oxidação do metanol, os resultados de DEMS indicam a formação de massa 44, ou seja, a oxidação ocorre via formação de intermediários fortemente adsorvidos. A obtenção da massa 60 indica que a oxidação do metanol ocorre em uma certa extensão via um mecanismo paralelo em que o ácido fórmico reage com o metanol formando metil-formiato (massa 60).

\section{Agradecimento} financeiro.

A. Oliveira Neto; M. Linardi; E. R. Gonzalez. The electro-oxidation of methanol on PtRu and PtMo particules supported on high-surface-area carbon.

Abstract: The electro-oxidation of methanol was studied on $\mathrm{Pt} / \mathrm{C}, \mathrm{PtRu} / \mathrm{C}$ and $\mathrm{PtMo} / \mathrm{C}$. These catalysts were prepared by the formic acid method developed in this laboratory.

The products of methanol oxidation were monitored by DEMS experiments.

The activity of the catalysts for the oxidation of methanol was studied by cyclic voltametry and cronamperometry.

Keywords: electrocatalysts; methanol oxidation; fuel cells.

\section{Referências bibliográficas}

[1] R. Parsons, T. Vandernoot, J. Electroanal. Chem. 257 (1988) 9.

[2] A. Hamnett. Catal. Today 38 (1997) 445.

[3] T. Zerihum, P. Grundler. J. Electroanal. Chem. 441 (1998)

57.

[4] A.V. Tripkovic, K.D. Popovic, R. R. Adzic. Russ. J. Electrochem. 31 (1995) 993.

[5] X. H. Xia, T. Iwasita, F. Ge. Electrochim. Acta 41 (1996) 711.

[6] A.V. Tripkovic, K. D. Popovic, R. R. Adzic. Electrochim. Acta. 44 (1998) 1145;

[7] S. SriramulU, T. D. Jarvi, E. M. Stuve. J. Electroanal. Chem. 467 (1999) 132.

[8] J. Prabhuram, R. Manoharan. J. Power Sources, 74 (1998) 54.

[9] R. A. Lampitt, L.P. L Carrette, M. P. Hogarth, A. E. Russell. J. Electroanal. Chem. 460 (1999) 80.

[10] A. Kabbabi, R. Faure, R; Durand, B. Beden, F. Hahn, J. M. Leger, C. Lamy. J. Electroanal. Chem. 444 (1998) 41.

[11] H. A. Gasteiger, N. M. Markovic, P. N Ross, E. J. Cairns. J. Electrochem. Soc. 141 (1994) 1795

[12] H. A. GASTEIGER, N. M. MARKOVIC, P. N. ROSS, E.
J. CAIRNS. Electrochim. Acta 39 (1994) 1825.

[13] H. A. Gasteiger, N. M. Markovic, P. N. Ross, E. J. Cairns, J .Phys. Chem. 97 (1993) 12020.

[14] F. Gloaguen, J.-M. Leger, C. Lamy, J. Appl. Eletrochem. 27 (1997) 1052.

[15] R. Ramkumar; S. Dheeenadayalan, R. Pattabiraman. J. Power Sources 69 (1997) 75.

[16] P. C. Biswas, Y. Nodasaka, M. Enyo, J. Appl. Eletrochem. 26 (1996) 30.

[17] T.D. Tran, S. H. Langer. Electrochim. Acta 38 (1993) 1551. [18] L. Liu, C. Pu, R. Viswanathan, Q. Fan, E. Liu, E. S.Smotkin. Eletrochim. Acta 43, (1998) 3657.

[19] C. T. Hable, M. S. Wrighton. Langmuir 9 (1993) 3284.

[20] G. J. K. Acres, J. C. Frost, G. A. Hards, R. J. Potter, T. R. Ralph, D. Thompsett, G. T. Burstein, G. J. Hutchings. Catal. Today 38 (1997) 393.

[21] T. Iwasita, H. Hoster, A. John-Anacker, W. F. Lin, W. Vielstich. Langmuir 16 (2000) 522.

[22] S. Swathijaran, Y. M. Mikhail. J. Electrochem. Soc. 138, (1991) 2105.

[23] W. Crzanowski, A. Wieckowski, W. Crzanowski. Langmuir 14 (1998) 1967.

[24] T J. Schmidt, M. Noeske, H. A. Gastaiger, R. J. Behm, P.Britz. J. Electrochem. Soc. 145 (1998) 925. 
[25] L. Liu, R. Viswanathan, R. Liu, E. S. Smotkin. Electrochem. and Solid.-State Lett. Electrochem. Solid-State Lett. 1 (1998) 123.

[26] Y. Morimoto, E. B. Yeager. J. Electroanal. Chem. 444 (1998) 95.

[27] G. Tremiliosi-Filho, H.Kim, W. Chrzanowski, A. Wieckowski, B. Grzybowska, P. Kulesza. J. Electroanal. Chem. 467 (1999 143.

[28] T. Frelink, W. Visscher, J. A. R. Vanveen. Surf. Sci. 335 (1995) 353.

[29] F. Richarz, B. Wohlmann, U. Vogel, H. Hoffschulz, K. Wandelt. Surf. Sci. 335, (1995) 361.

[30] T. Frelink, W. Visscher, J. A. R.Vanveen. Eletrochim. Acta. 39 (1994) 1871.

[31] A. S. Aricó, Z. Poltarzewski, H. Kim, A. Morana, N. Giordano, V. Antonucci. J. Power Sources 55 (1995) 159.

[32] K. L. Ley, R. Liu, C. Pu, Q. Fan, N. Leyarovska, C. Segre, Smotkin, E. S. J. Electrochem. Soc. 144 (1997) 1543. [33] B. Gurau, R. Viswanathan, R. Liu, T. J. Lafrenz, K. L. Ley, E. S. Smotkin, E. Reddington, A. Sapienza, B. C. Chan, T. E. Mallouk, S. Sarangapani. J. Phys. Chem. B 102 (1998) 9997.

[34] W.T. Napporn, J. -M. Léger, C. Lamy. J. Electroanal. Chem. 408 (1996) 141.

[35] M. Gotz, H. Wendt. Electrochim. Acta 43 (1998) 3637. [36] A. S. Arico, H. Kim, A. K. Shukla, M. K. Ravikumar, V. Antonucci, GIORDANO. Electrochim. Acta 39 (1994), 691.

[37] A. Hamnett, J. Kennedy. Electrochim.Acta 33 (1988)
1613.

[38] T Iwasita, F. C. Nart, W. Vielstich. Berlin Bun. Fur Phys. Chem. 94 (1990) 1030.

[39] T. Frelink, W. Visscher, A. P. Cox, J. A. R. Vanveen. Electrochim. Acta. 40 (1995) 1537.

[40] T. Iwasita, W. Vielstich. Progress in the study of the methanol oxidation by In-situ, Ex-situ and On-line methods. Weinheim,VCH,1990. v.1, p.127-170.(Advances in Electrochemical Sciences and Engineering).

[41] J. Munk, P. A. Christensen, A. Hamnett, E. Skou. J. Electroanal. Chem. 401 (1996) 215.

[42] K. Kinoshita. J. Electrochem. Soc. 137 (1990) 845.

[43] A. K. Shukla, M. K. Ravikumar, A. S. Arico, G. Candiano, V. Antonucci, N. Giordano, A. Hamnett. J. Appl. Electrochem. 25 (1995) 528.

[44] M. Shibata, N. Furuya. J. Electroanal. Chem. 229 (1989) 217.

[45] A. Oliveira-Neto, J. Perez, W. T. Napporn, E. A. Ticianelli, E. R. Gonzalez. Electrooxidation of methanol on binary platinum based catalysts. IN:Workshop Electrocatalysis in Indirect and Direct Methanol PEM Fuel Cells. IIIrd International Symposium in Electrocatalysi. Portoroz, Slovenia, 1999, p.83-6.

[46] J. P. I. Souza. Eletro-oxidação de metanol e etanol sobre eletrodepósitos de Pt,Rh,PtRh e PtRu. Um estudo de eletrocatálise usando técnicas de FTIR in situ e DEMS online. São Carlos,1999. 157p. Tese (Doutorado)- IQSC Instituto de Química de São Carlos,Universidade de São Paulo. 